\title{
Incidence and detection of high microsatellite instability in colorectal cancer in a Chinese population: a meta-analysis
}

\author{
Congjun Zhang ${ }^{1 \#}$, Hongguang Ding ${ }^{2 \#}$, Shijun Sun ${ }^{3}$, Zhonghua Luan ${ }^{4}$ Guoyan Liu ${ }^{5,6,7}, \mathrm{Zhi} \mathrm{Li}^{8}$ \\ ${ }^{1}$ Department of Oncology, The First Affiliated Hospital of Anhui Medical University, Hefei, China; ${ }^{2}$ Department of General Surgery, Qingdao \\ Municipal Hospital, Qingdao, China; ${ }^{3}$ The Molecular Diagnostic Center, Zhongshan City People's Hospital, Zhongshan, China; ${ }^{4}$ Department of \\ Pathology, Yuncheng Central Hospital, Yuncheng, China; Department of Gastrointestinal Surgery, Zhongshan Hospital of Xiamen University, \\ Xiamen, China; ${ }^{6}$ Institute of Gastrointestinal Oncology, Medical College of Xiamen University, Xiamen, China; ${ }^{7}$ School of Pharmaceutical Sciences \\ Xiamen University, Xiamen, China; ${ }^{8}$ Department of Pathology, Guangdong Provincial Peoples' Hospital, Guangzhou, China \\ Contributions: (I) Conception and design: Z Li, G Liu; (II) Administrative support: C Zhang, H Ding; (III) Provision of study materials or patients: S \\ Sun; (IV) Collection and assembly of data: Z Luan; (V) Data analysis and interpretation: C Zhang, H Ding; (VI) Manuscript writing: All authors; (VII) \\ Final approval of manuscript: All authors. \\ \#These authors contributed equally to this work. \\ Correspondence to: Zhi Li. Department of Pathology, Guangdong Provincial Peoples' Hospital, 106 Zhongshan Er Road, Guangzhou 510080, China. \\ Email: lizhi_sysu@126.com; Guoyan Liu. Department of Gastrointestinal Surgery, Zhongshan Hospital of Xiamen University, 205 Hubin South \\ Road, Xiamen 361004, China. Email: liuguoyan@xmu.edu.cn.
}

Background: The 4 most common types of DNA mutations in tumors are single-nucleotide variations, insertion-deletion, fusion, and copy number variations. This is followed by microsatellite instability (MSI), which is known to trigger the development of MSI-high (MSI-H) cancer and is responsible for 300,000 new cases of cancer per year in China. We aim to conduct a meta-analysis based on a comparison between the positive rates of the National Cancer Institute (NCI) panel (also known as 2B3D NCI panel) and mononucleotide panels for the diagnosis of MSI in the Chinese population.

Methods: In the present meta-analysis, we searched the PubMed, Embase, Web of Science, CNKI, Wanfang, CQVIP, and CBM databases. MSI diagnosis studies by PCR and capillary electrophoresis were included to compare the incidence of MSI-H in colorectal cancer obtained from panels with different microsatellite markers. Egger's bias test was used to assess risk of bias.

Results: Seventeen articles were included, which used the Newcastle-Ottawa Scale (NOS) scale for quality evaluation. The NOS scores of the included documents were $\geq 7$ points, and the quality of the documents met the requirements. The incidence of MSI-H detected by the 2B3D NCI panel was $13.5 \%$ [95\% confidence interval (CI): $10.8-16.4, \mathrm{I}^{2}=52.321 \%, \mathrm{P}=0.026, \mathrm{n}=10$ studies including 2,681 participants], the incidence of MSI-H detected by the mononucleotide panels was $10.6 \%$ (95\% CI: 7.1-14.7, $\mathrm{I}^{2}=81.147 \%, \mathrm{P}=0.000, \mathrm{n}=7$ studies including 3,249 participants). This indicates that, in the Chinese population, the 2B3D NCI panel can detect $27.4 \%$ more MSI-H cancers than the mononucleotide panels, $54.7 \%$ more MSI-H cancers than the panel of 6 mononucleotides, and its sensitivity is comparable to that of Promega.

Conclusions: The findings of the meta-analysis demonstrated that, using the 2B3D NCI panel for MSI detection can avoid the underestimation of the incidence MSI-H in colorectal cancer and can be considered the most suitable panel for MSI detection in the Chinese population. The inclusion of only published data might be a potential source of publication bias.

Keywords: 2B3D NCI panel; microsatellite instability (MSI); MSI-high (MSI-H) cancer; colorectal cancer (CRC)

Submitted Sep 11, 2020. Accepted for publication Dec 04, 2020.

doi: 10.21037/jgo-20-487

View this article at: http://dx.doi.org/10.21037/jgo-20-487

(C) Journal of Gastrointestinal Oncology. All rights reserved. 


\section{Introduction}

The 4 most common types of DNA mutations in tumors are single-nucleotide variations, insertion-deletion, fusion, and copy number variations, which are relatively simple mutations that typically cause abnormalities in the function of 1-2 genes and signal transductions in their pathways. Microsatellite instability (MSI) is the 5 th most common mutation in tumors and is defined as an alteration of any length due to either the insertion or deletion of repeat units, named "microsatellites", within a tumor tissue when compared to normal tissue (1). MSI is a hypermutable phenotype caused by the loss of function of the DNA mismatch repair (MMR) system, which is responsible for repairing DNA mismatch errors that occur during DNA replication. MSI is a more complex mutation. As microsatellites are distributed throughout the entire genome, MSI in different genes will result in the dysfunction of these genes and will affect multiple signaling pathways associated with cancer, leading to the occurrence and progression of MSI-high (MSI-H) tumors.

MSI occurs in various types of cancer, including colorectal, endometrial, gastric, small bowel, ovarian, and prostate cancer. The incidence of MSI-H varies among different cancers, and has been found to be highest in endometrial, gastric, and colorectal cancers $(33 \%, 22 \%$, and $13 \%$, respectively) (2). It also varies among different populations in the same type of cancer; for example, for colorectal cancer, the incidence of MSI-H is highest among Egyptians (37\%) and African Americans (20-45\%), but lowest among Europeans and Americans (8-20\%) (3).

Colorectal cancer is one of the most common cancers in the Chinese population, the instance rate and the mortality rate of colorectal cancer keep increasing (4). MSI diagnosis plays a pivotal role in the precision diagnosis and treatment of colorectal cancer and other solid tumors, as reflected principally in the following four aspects: (I) Universal MSI/ MMR testing is recommended in all diagnosed patients with colorectal cancer for Lynch syndrome screening; (II) MSI-H tumors have better prognosis than microsatellite stable (MSS) tumors; (III) colorectal cancer patients with MSI-H at stage II cannot benefit from 5-flurouracil chemotherapy; and (IV) MSI-H or deficient MMR (dMMR) tumors have been shown to be effective in immunotherapy in multiple solid tumors. Therefore, MSI detection is critical for solid tumors, especially in colorectal cancer.

Currently, MSI diagnostic approaches used for human tumors mainly include the polymerase chain reaction (PCR)-based method and next-generation sequencing.
Multiplex fluorescence PCR followed by capillary electrophoresis fragment analysis is considered as the international gold standard approach for identifying MSI status in cancer, because of its high-throughput, higher sensitivity and reproducibility. The most critical factor influencing the sensitivity of MSI diagnosis is the loci of the microsatellite markers used. Different MSI diagnostic panels have been proposed for the sensitive detection of MSI, including the 2B3D NCI panel, which has been the gold standard panel for MSI diagnosis since 1997. The NCI panel consists of 2 mononucleotide repeat markers (BAT-25 and BAT-26) and 3 dinucleotide repeat markers (D2S123, D5S346, and D17S250). The classification criteria for MSI status are as follows. When compared to normal tissue, tumors with $\geq 2$ unstable microsatellite markers are defined as MSI-H, those with only 1 unstable microsatellite marker are defined as MSI-low(MSI-L), and those with all 5 stable microsatellite markers are defined as MSS (1). According to previously published studies on Caucasians, the sensitivity of dinucleotide loci showed lower sensitivity than mononucleotide loci; therefore, panels composed of mononucleotides are also widely used in MSI diagnosis, such as the pentaplex panel of 5 mononucleotides (BAT25, BAT-26, NR-21, NR-24, and MONO-27), which is commercially available from manufacturers, such as Promega (Madison, WI, USA).

However, a comparison of positive rates between the 2B3D NCI panel and mononucleotide panels is lacking for the Chinese or East Asian population. No consensus has been reached on the selection of MSI diagnostic panels in Chinese population due to lack of data support. In the present study, we used the PCR-based approach to analyze different panels for the diagnosis of MSI in the East Asian population, and conducted a meta-analysis based on a comparison between the positive rate of the $2 \mathrm{~B} 3 \mathrm{D}$ and mononucleotide panels, and tried to find out a panel with better sensitivity in Chinese population in order to provide a clearer guidance for panel selection.

We present the following article in accordance with the PRISMA reporting checklist (available at http://dx.doi. org/10.21037/jgo-20-487).

\section{Methods}

\section{Literature inclusion and exclusion criteria}

The inclusion criteria for the present study were as follows: (I) the study type was a cohort study; (II) a definite diagnosis of colorectal cancer; and (III) articles published in Chinese 
or English. The exclusion criteria were as follows: (I) duplicate publications; (II) review articles, animal experiments, and case reports; (III) studies without full text, studies with incomplete information, or studies where data extraction was not possible; (IV) studies without available incidence of MSI-H; (V) articles without using PCR and capillary electrophoresis; (VI) articles published previous to 2000.

\section{Search strategy}

In the present systematic review and meta-analysis, we searched the PubMed, Embase, Web of Science, CNKI, Wanfang, CQVIP, and CBM databases. The search terms were as follows: "colorectal neoplasms", "colorectal cancer", "microsatellite instability", "MSI", "Chinese”, and "East Asian".

The search strategy was as follows: $((()(()(($ “colorectal neoplasms”[MeSH] ) or (colorectal neoplasm[title/abstract])) OR (neoplasm, colorectal[title/abstract])) OR (colorectal carcinoma[title/abstract])) OR (colorectal carcinomas[title/ abstract])) OR (colorectal cancer[title/abstract])) OR (colorectal cancers[title/abstract])) OR (colorectal tumors[title/abstract])) OR (colorectal tumor[title/ abstract])) AND ((microsatellite instability[title/abstract]) OR (MSI[title/abstract]))) AND ((Chinese[title/abstract]) OR (East Asian[title/abstract])).

\section{Literature screening and data extraction}

The literature search, screening, and information extraction were completed by 2 researchers independently. If consensus could not be reached, a 3 rd researcher was consulted. Data extraction included author, year, type of study, sample size, and clinical indicators.

\section{Literature quality assessment}

Two researchers independently used Newcastle-Ottawa Scale (NOS) to evaluate the quality of the literature. If consensus could not be reached, a 3 rd researcher was consulted. NOS includes 4 items (4 points) for "selection of research participants"; 1 item ( 2 points) for "comparability between groups" and 3 items (3 points) for "result measurement", with a total score of 9 points and $\geq 7$ points high-quality literature, $<7$ is divided into lower-quality literature.

\section{Statistical analysis}

Stata 15.1 (StataCorp, College Station, TX, USA) was used for the single-group rate analysis on the data. $\mathrm{I}^{2}$ was used to evaluate heterogeneity. If the heterogeneity test showed $\mathrm{P} \geq 0.1$ and $\mathrm{I}^{2} \leq 50 \%$, this indicated that the study had homogeneity. The fixed-effects model was used for the combined analysis. If $\mathrm{P}<0.1$ and $\mathrm{I}^{2}>50 \%$, this indicated that the study had heterogeneity. Sensitivity analysis or subgroup analysis was used to find the source of heterogeneity, the sensitivity analysis was performed by excluding the literature one by one. If the heterogeneity was still significant, the random-effects model was used or descriptive analysis. When the number of documents included in a single outcome index was $>10$, the publication bias of each index was analyzed using a funnel chart and Egger's bias test.

\section{Results}

\section{Literature search results}

In total, 1,832 articles were obtained by searching the PubMed, Embase, Web of Science databases, CNKI, Wanfang, CQVIP, and CBM databases. After excluding duplicate articles, 926 articles remained. Article abstracts were browsed and a further 574 articles were included. After reading the full text, we obtained a further 17 articles that could be used in meta-analysis (Figure 1) (Table 1).

\section{Baseline characteristics and quality assessment of the included studies}

The included 17 articles used the NOS scale for quality evaluation. The NOS scores of the included documents were $\geq 7$ points, and the quality of the documents met the requirements (Table 2).

\section{Results of meta-analysis}

The incidence of MSI-H detected by the 2B3D NCI panel was $13.5 \%$ [ $95 \%$ confidence interval (CI): $10.8-$ $16.4, \mathrm{I}^{2}=52.321 \%, \mathrm{P}=0.026, \mathrm{n}=10$ studies including 2,681 participants], the incidence of MSI-H detected by Promega was $12.9 \%$ (95\% CI: $8.3-18.3, \mathrm{I}^{2}=60.891 \%, \mathrm{P}=0.053$, $\mathrm{n}=4$ studies including 640 participants), the incidence of MSI-H detected by 6 mononucleotide markers was $7.7 \%$ (95\% CI: $4.7-11.2, \mathrm{I}^{2}=61.685 \%, \mathrm{P}=0.074, \mathrm{n}=4$ studies including 2,609 participants), the incidence of MSI-H detected by mononucleotide panels was $10.6 \%$ (95\% CI: 7.1-14.7, $\mathrm{I}^{2}=81.147 \%, \mathrm{P}=0.000, \mathrm{n}=7$ studies including 3,249 participants) (Figures 2-5). 


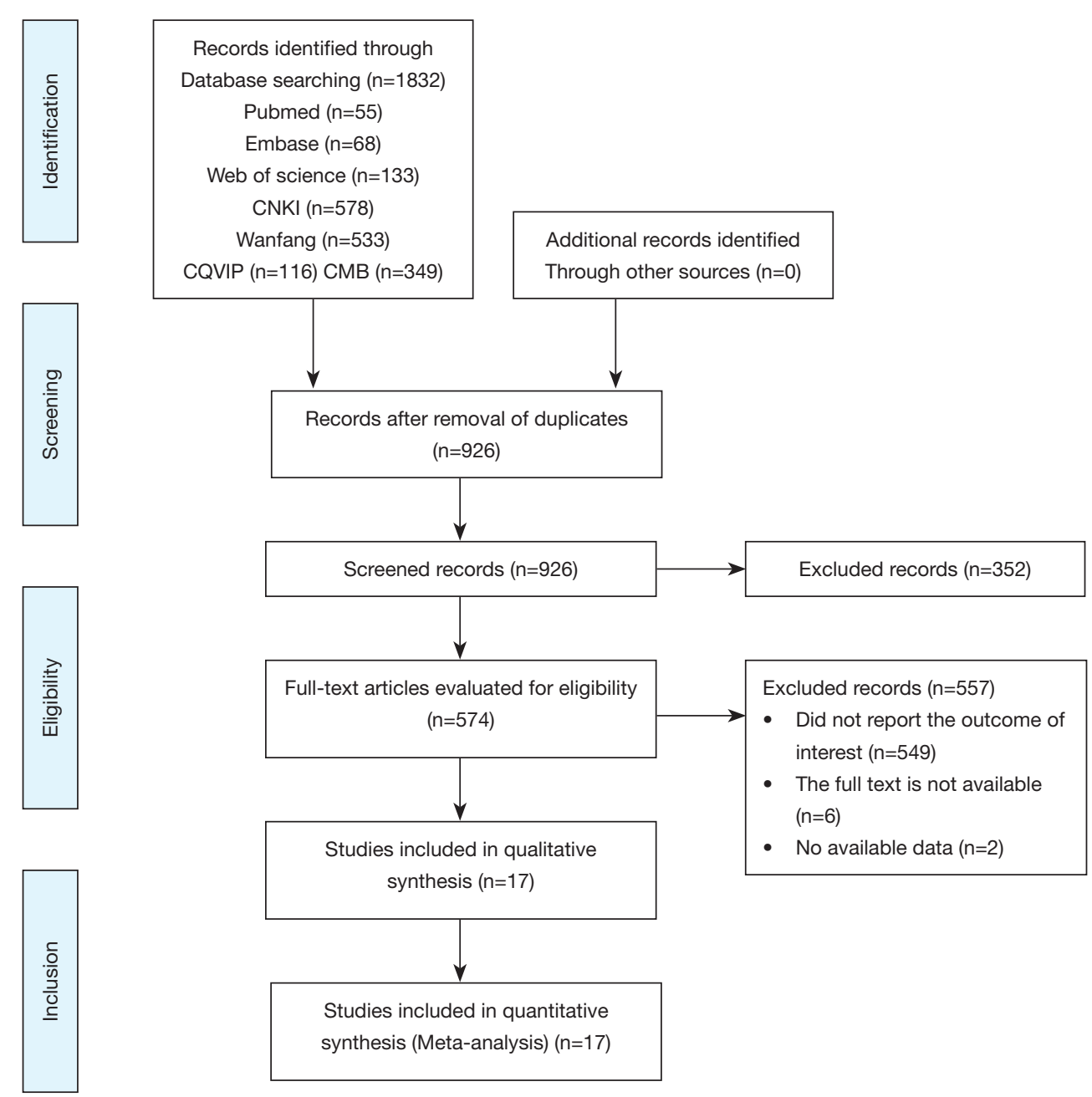

Figure 1 Flow diagram for selection of studies.

\section{Publication bias}

As shown in Figure 6, based on the results of the Egger's bias test $(\mathrm{P}=0.274,>0.05)$, there was no obvious publication bias in the present study, and the funnel chart was symmetrical.

\section{Sensitivity analysis}

No studies that had a significant impact on the results were found, suggesting that the results of the present study were relatively stable.

\section{Discussion}

There were approximately 4,292,000 new cancer cases in China in 2015. Based on the incidence of MSI-H in different types of cancer, we estimate that 300,000 new
MSI-H cancer cases per year in the Chinese population; this value is comparable to breast cancer $[272,400]$ and colorectal cancer $[376,300](2,4)$. Despite the severe burden of MSI-H cancer, its role in clinical diagnosis and treatment has been underreported.

The reasons affecting MSI-H incidence vary. First, MSI-H incidence among populations differs; for example, in colorectal cancer, the incidence of MSI-H is highest among Egyptians (37\%) and African Americans (20-45\%), but lowest among Europeans and Americans (8-20\%) (3). In comparison MSI-H, incidence in Chinese patients is about $4.5-15 \%$. Second, MSI-H incidence varies among different stages. MSI-H is more frequent in stage II colorectal cancer (20\%) than stage III (12\%), and is rare in stage IV (4\%) (3). Third, the incidence obtained from different methods or panels composed with different microsatellite markers. The 
Table 1 Baseline characteristics of the included studies

\begin{tabular}{|c|c|c|c|c|c|c|}
\hline Author & Country & search type & Cases (n) & Age (years) & Sex (male/female) & Panel \\
\hline Jin et al., 2007 (6) & China & Cohort & 110 & $64.0(46.0-94.0)$ & $68 / 42$ & 2B3D \\
\hline Yang et al., 2007 (7) & China & Cohort & 105 & 60.5 (13.0-94.0) & $66 / 39$ & 2B3D \\
\hline Jin et al., 2010 (8) & China & Cohort & 150 & $53.2(23.0-85.0)$ & $73 / 77$ & 2B3D \\
\hline Peng et al., 2015 (10) & China & Cohort & 75 & $\mathrm{~N} / \mathrm{A}$ & $44 / 31$ & 2B3D \\
\hline Zhou et al., 2015 (11) & China & Cohort & 80 & $\mathrm{~N} / \mathrm{A}$ & $\mathrm{N} / \mathrm{A}$ & 2B3D \\
\hline Yan et al., 2016 (12) & China & Cohort & 182 & $\mathrm{~N} / \mathrm{A}$ & $\mathrm{N} / \mathrm{A}$ & 2B3D \\
\hline Zheng et al., 2018 (13) & China & Cohort & 245 & $\mathrm{~N} / \mathrm{A}$ & $139 / 106$ & 2B3D \\
\hline Bai et al., 2019 (14) & China & Cohort & 72 & $\mathrm{~N} / \mathrm{A}$ & N/A & Promega \\
\hline Jiang et al., 2019 (15) & China & Cohort & 213 & $59.0(19.0-92.0)$ & $118 / 95$ & Promega \\
\hline Huang et al., 2010 (16) & China & Cohort & 298 & 54.6 & $184 / 114$ & Promega \\
\hline Li et al., 2018 (17) & China & Cohort & 240 & $\mathrm{~N} / \mathrm{A}$ & $153 / 87$ & 6 mononucleotide markers \\
\hline Wang et al., 2019 (18) & China & Cohort & 40 & $\mathrm{~N} / \mathrm{A}$ & $24 / 16$ & 6 mononucleotide markers \\
\hline Song et al., 2020 (19) & China & Cohort & 2,356 & N/A & $1,437 / 919$ & 6 mononucleotide markers \\
\hline
\end{tabular}

$\mathrm{N} / \mathrm{A}$, not available.

Table 2 Quality assessment

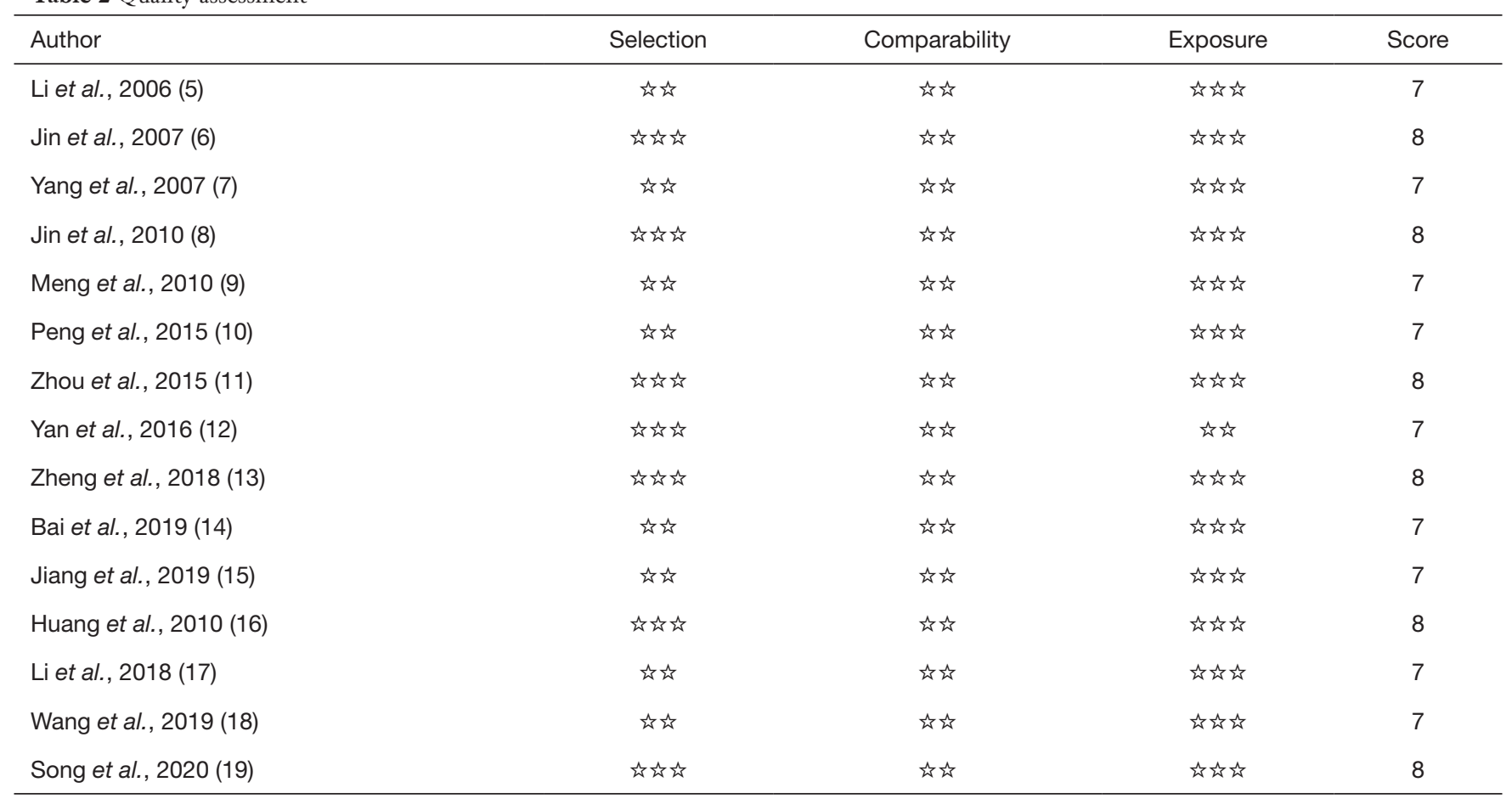




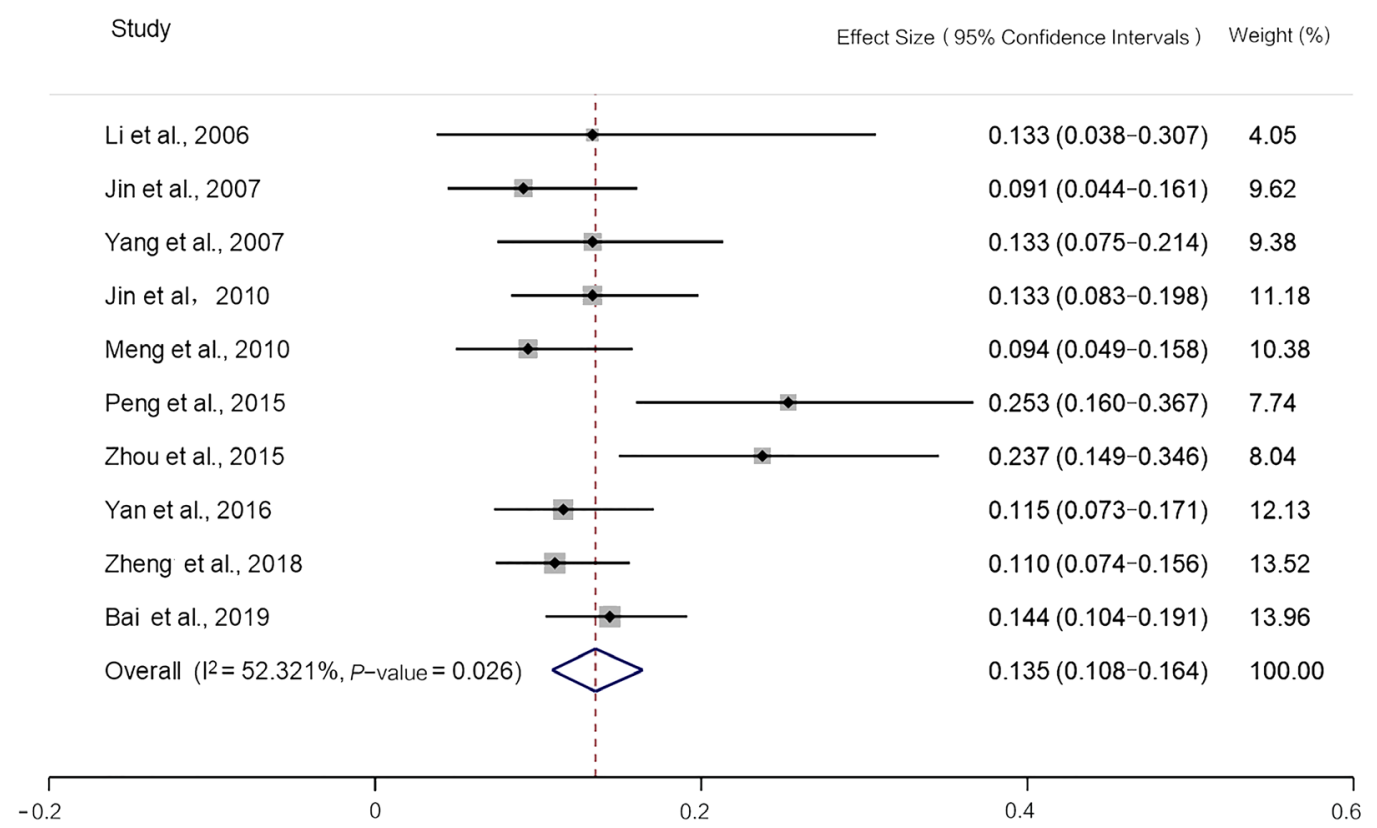

Figure 2 Forest plot of the incidence of microsatellite instability-high, detected by the 2B3D National Cancer Institute panel.

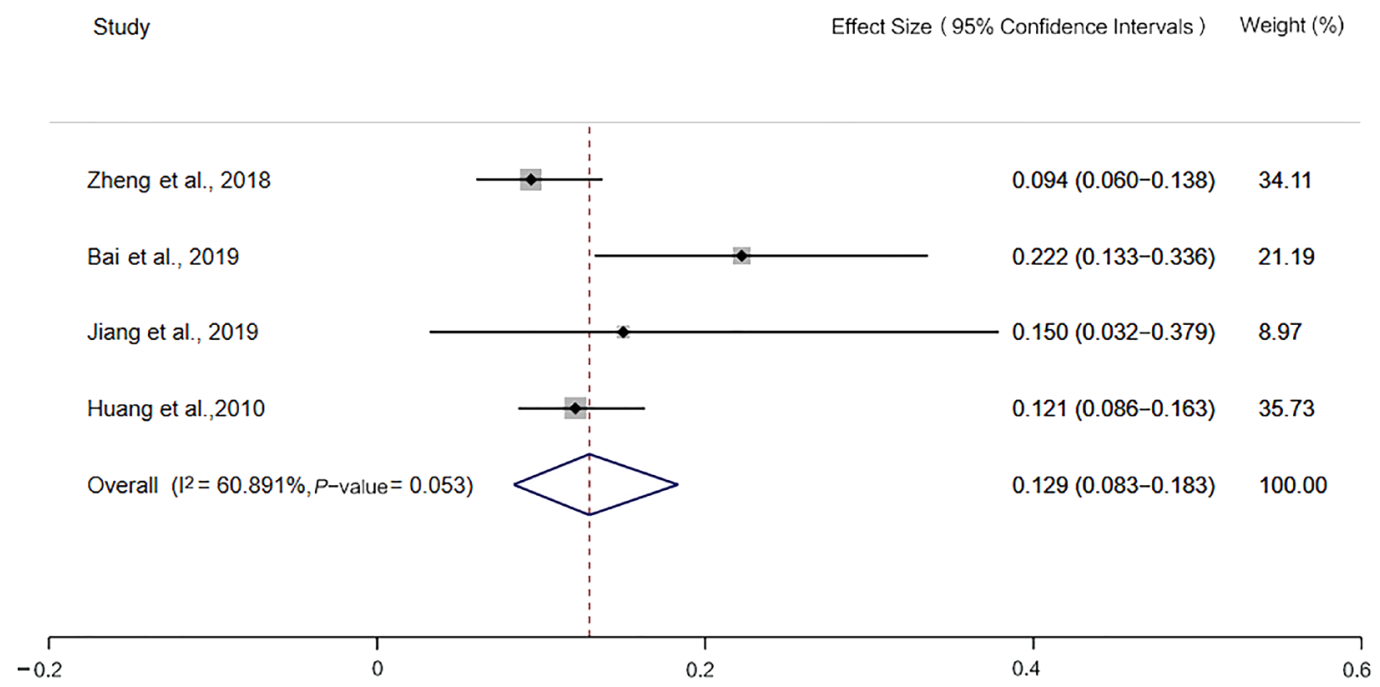

Figure 3 Forest plot of the incidence of microsatellite instability-high, detected by Promega.

PCR-based method, recognized as the gold standard for MSI detection, has higher sensitivity and specificity than other methods. However, the incidence obtained from panels using different microsatellite markers would also be different. To the best of our knowledge, the present study is the first to compare the incidence of MSI-H in colorectal cancer obtained from panels with different microsatellite markers. The incidence of MSI-H detected by the $2 \mathrm{~B} 3 \mathrm{D}$ NCI panel in the present study was $13.5 \%$ (95\% CI: $10.8-16.4, \mathrm{I}^{2}=52.321 \%, \mathrm{P}=0.026, \mathrm{n}=10$ studies including 2,681 participants), the incidence of MSI-H detected by mononucleotide panels was $10.6 \%$ (95\% CI: 7.1-14.7, $\mathrm{I}^{2}=81.147 \%, \mathrm{P}=0.000, \mathrm{n}=7$ studies including 3,249 participants). Therefore, in the Chinese population, the 


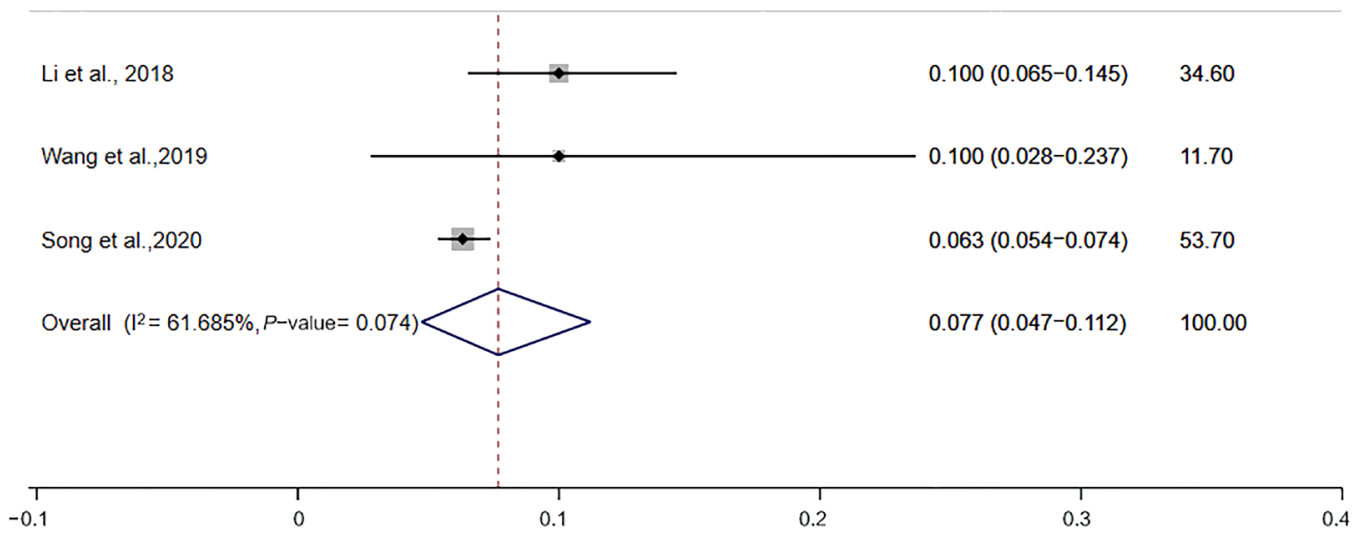

Figure 4 Forest plot of the incidence of microsatellite instability-high, detected by 6 mononucleotide markers.

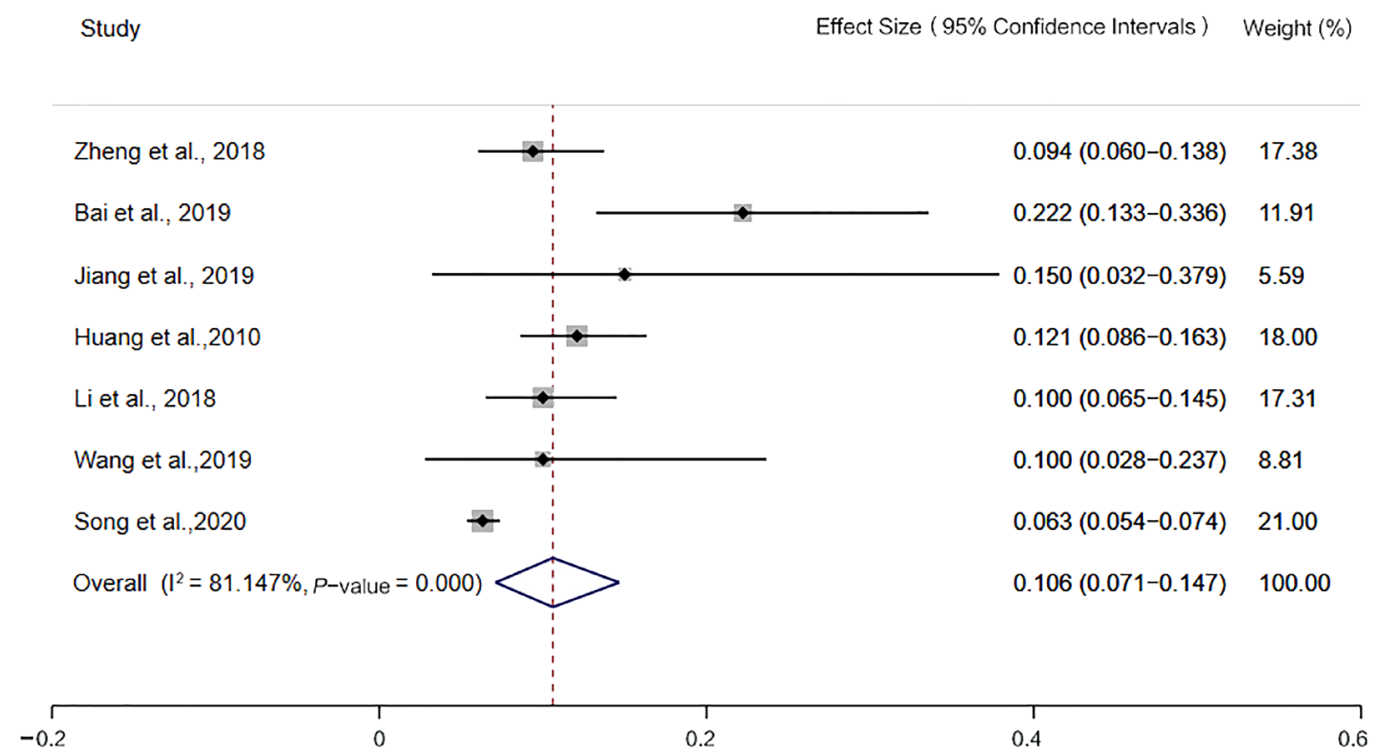

Figure 5 Forest plot of the incidence of microsatellite instability-high, detected by mononucleotide markers.

2B3D NCI panel can detect 27.4\% more MSI-H cancers than mononucleotide panels, $54.7 \%$ more MSI-H cancers than the panel of 6 mononucleotides, and its sensitivity is comparable to that of Promega.

The incidence obtained from different combinations of microsatellite markers differs greatly, as each microsatellite marker has a different sensitivity. For example, the BAT25 and BAT-26 mononucleotide markers are considered as the most sensitive markers in MSI detection, whereas the sensitivity of NR-24 is lower than the former 2 markers, and
D2S123 is the most sensitive dinucleotide marker. In addition, an increase in the number of microsatellite markers does not necessarily increase the sensitivity and accuracy of MSI detection. If microsatellite markers with lower sensitivities are included in the combination, the overall sensitivity of the MSI detection panel will decrease significantly (14).

In Caucasians, mononucleotide markers have been found to be more sensitive and specific than dinucleotide markers for MSI detection when identifying hereditary non-polyposis colorectal cancer (HNPCC) (20). However, 


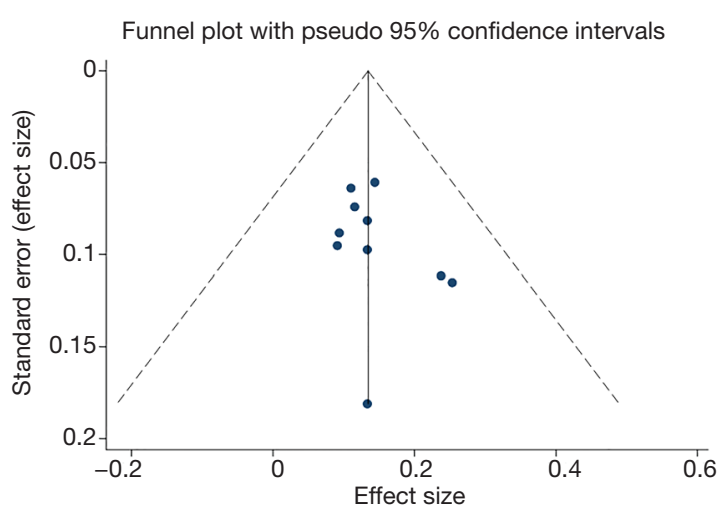

Figure 6 Funnel plot for testing publication bias.

in the Chinese population, we obtained the opposite result. The potential reason might be the instability of the 3 dinucleotide markers is an earlier event in tumorigenesis in the Chinese population than that of the mononucleotides markers other than BAT-25 and BAT-26. Currently, the detection of MSI is no longer limited to HNPCC/Lynch syndrome screening. With recent advances in immune checkpoint inhibitors, the value of MSI detection has focused more on predicting responsiveness of the PD-1/ PD-L1 and CTLA-4 inhibitors in solid cancers. Therefore, in the present study, only sporadic colorectal cancers were included in the meta-analysis. Nash et al. demonstrated that, in HNPCC, the sensitivities of the BAT-25 and BAT26 mononucleotides were high, whereas they were lower in sporadic colorectal cancers. On the contrary, the sensitivity of dinucleotide markers was higher in sporadic colorectal cancers than in HNPCC (21). Yang et al. confirmed this result in a Chinese population (7), which could also explain the higher sensitivity of dinucleotide markers compared with mononucleotide markers in the present study.

A number of studies have suggested that the occurrence of MSI-H varies cross different populations (3); therefore, microsatellite marker selection needs to be supported by the clinical data from Chinese population.

The National Comprehensive Cancer Network guidelines and Chinese Society of Clinical Oncology guidelines for colorectal cancers recommend using the PCR-based method for MSI detection, and the latter explicitly recommends the 2B3D NCI panel for determining MSI status. In a study of 245 East Asian patients with colorectal cancer, the 2B3D NCI panel showed better sensitivity $(89.4 \%)$ than Promega (71.4\%) for detecting MSI (13). Similar to this result, in another study of 271 East
Asian patients with colorectal cancer, the 2B3D NCI panel was also compared with Promega, with the 2B3D NCI panel demonstrating higher sensitivity (87.18\%) compared with Promega (77.78\%) (14). These 2 studies demonstrated that the 2B3D NCI panel could be the most suitable panel for MSI detection in an East Asian population $(13,14)$.

The present study has some limitations. First, as it was a single-arm meta-analysis, we were not able to evaluate statistical significance. Second, the inclusion of only published studies might be a potential source of publication bias. Third, grey literature may have reported negative findings. Therefore, our results should be interpreted with caution.

The present meta-analysis demonstrated that the 2B3D NCI panel can detect 27.4\% more MSI-H cancers than mononucleotide panels in the Chinese population. Therefore, using the 2B3D NCI panel for MSI detection can avoid the underestimation of the incidence of colorectal cancer and can be considered the most suitable panel for MSI detection in the Chinese population. Future studies with larger sample sizes are needed to confirm this result.

\section{Acknowledgments}

Funding: None.

\section{Footnote}

Reporting Checklist: The authors have completed the PRISMA reporting checklist. Available at http://dx.doi. org/10.21037/jgo-20-487

Conflicts of Interest: All authors have completed the ICMJE uniform disclosure form (available at http://dx.doi. org/10.21037/jgo-20-487). The authors have no conflicts of interest to declare.

Ethical Statement: The authors are accountable for all aspects of the work in ensuring that questions related to the accuracy or integrity of any part of the work are appropriately investigated and resolved.

Open Access Statement: This is an Open Access article distributed in accordance with the Creative Commons Attribution-NonCommercial-NoDerivs 4.0 International License (CC BY-NC-ND 4.0), which permits the noncommercial replication and distribution of the article with the strict proviso that no changes or edits are made and the original work is properly cited (including links to both the 
formal publication through the relevant DOI and the license). See: https://creativecommons.org/licenses/by-nc-nd/4.0/.

\section{References}

1. Boland CR, Thibodeau SN, Hamilton SR, et al. A National Cancer Institute Workshop on Microsatellite Instability for Cancer Detection and Familial Predisposition: Development of International Criteria for the Determination of Microsatellite Instability in Colorectal Cancer. Cancer Res 1998;58:5248-57.

2. Wang C, Gong J, Tu TY, et al. Immune profiling of microsatellite instability-high and polymerase epsilon (POLE)-mutated metastatic colorectal tumors identifies predictors of response to anti-PD-1 therapy. J Gastrointest Oncol 2018;9:404-15.

3. Vilar E, Gruber S. Microsatellite instability in colorectal cancer-the stable evidence. Nat Rev Clin Oncol 2010;7:153-62.

4. Chen W, Zheng R, Baade P, et al. Cancer statistics in China, 2015. CA Cancer J Clin 2016;66:115-32.

5. Li X, Liu QH. Study on Microsatellite Instability in Colorectal Cancer Patients with Familial Predisposition. J China Med Univ 2006;37:410-08.

6. Jin HY, Lai RS, Ding YJ, et al. Detection of microsateilite instability in colorectal cancer by fluorescence mulitplex polymerase chain reaction and its clinical value. Zhonghua Wei Chang Wai Ke Za Zhi 2007;10:217.

7. Yang BL, Gu YF. Microsatellite instability in sporadic colorectal cancer and its relationship with clinicopathological features. World Chinese Journal of Digestology 2007;15:1160-4.

8. Peng J, Xiao-ming M, Jian-qiu S, et al. Clinicopathological Features of Non-familial Colorectal Cancer with Highfrequency Microsatellite Instability. Chin Med Sci J 2010;25:228-32.

9. Meng WJ, Wang L, Yu YY, et al. Significance of microsatellite instability in sporadic stage II and III rectal cancer. Chongqing Med J 2010;39:2420-6.

10. Peng JL, Tang T, Ye ZL, et al. The relationship of microsatellite instability state with loss of mismatch repair proteins and clinical pathological characteristics in sporadic colorectal cancers. Chin J Cancer Biother 2015;22:479-83.

11. Zhou LY, Wan MZ, Liu YP, et al. Assessment of Microsatallite Instability in Colorectal Carcinoma: A Comparison Between Immunohistochemistry and PCR Method. Cancer Res Prev Treat 2015;42:1231-4.

12. Yan WY, Hu J, Xie L, et al. Prediction of biological behavior and prognosis of colorectal cancer patients by tumor MSI/MMR in the Chinese population. Onco Targets Ther 2016;9:7415-24.

13. Zheng J, Huang B, Nie X, et al. The clinicopathological features and prognosis of tumor MSI in East Asian colorectal cancer patients using NCI panel. Future Oncol 2018;14:1355-64.

14. Bai W, Ma J, Liu Y, et al. Screening of MSI detection loci and their heterogeneity in East Asian colorectal cancer patients. Cancer Med 2019;8:2157-66.

15. Jiang N. Relationship between microsatellite instability and clinicopathological features and prognosis in sporadic colorectal cancer. Acta Universitatis Medicinalis Anhui 2019;54:139-42.

16. Huang YQ, Yuan Y, Ge WT, et al. Comparative features of colorectal and gastric cancers with microsatellite instability in Chinese patients. J Zhejiang Univ Sci B 2010;11:647-53.

17. Li W, Li H, Liu R, et al. Comprehensive Analysis of the Relationship Between RAS and RAF Mutations and MSI Status of Colorectal Cancer in Northeastern China. Cell Physiol Biochem 2018;50:1496-509.

18. Wang Z, Tang XL, Wu XQ, et al. Mismatch Repair status between primary colorectal tumor and metastatic tumor, a retrospective consistent study. Biosci Rep 2019;39:BSR20190730.

19. Song Y, Wang L, Ran W, et al. Effect of Tumor Location on Clinicopathological and Molecular Markers in Colorectal Cancer in Eastern China Patients: An Analysis of 2,356 Cases. Front Genet 2020;11:96.

20. Umar A, Boland CR, Terdiman JP, et al. Revised Bethesda Guidelines for Hereditary Nonpolyposis Colorectal Cancer (Lynch Syndrome) and Microsatellite Instability. JNCI 2004;96:261-8.

21. Nash GM. Automated, multiplex assay for high-frequency microsatellite instability in colorectal cancer. J Clin Oncol 2003;21:3105-12.
Cite this article as: Zhang C, Ding H, Sun S, Luan Z, Liu G, $\mathrm{Li} Z$. Incidence and detection of high microsatellite instability in colorectal cancer in a Chinese population: a meta-analysis. J Gastrointest Oncol 2020;11(6):1155-1163. doi: 10.21037/jgo-20-487 